\title{
APPLICATIONS OF THE THEORY OF INTERACTING CONTINUA TO THE DIFFUSION OF A FLUID THROUGH A NON-LINEAR ELASTIC MEDIA
}

\author{
JOHN JIN-JAU SHI \\ Rocketdyne, Division of Rockwell International, Canoga Park, CA 91304, U.S.A
}

and

K. R. RAJAGOPAL and A. S. WINEMAN

Department of Mechanical Engineering and Applied Mechanics. The University of Michigan. Ann Arbor, MI 48109, U.S.A.

\begin{abstract}
The theory of interacting continua is applied to the problem of diffusiion of a fluid through a non-linear elastic layer and a hollow sphere. Using methods which are by now standard in continuum mechanics expressions and restrictions are derived from a thermodynamic standpoint for the partial stresses for the fluid and solid and the diffusive body force. In order to obtain detailed solutions to specific boundary value problems a choice of a particular form for the free energy function for the mixture is made based on statistical theory. To simplify the problem, we assume that the fluid in question is ideal. The difficulties inherent to a clear definition of the boundary conditions for the partial stresses are overcome by the use of the Flory-Huggins equation. Two specific examples are considered. The first is the problem of diffusion through a stretched layer and second is diffusion through a spherical shell. Results of the numerical solution enable the construction of the pressure difference-flux relations, which have been shown to be in good agreement with experimental data.
\end{abstract}

\section{INTRODUCTION}

IN THE classical approaches to the study of diffusion of a fluid through a solid, such as Fick's law [1] and Darcy's law [2], the solid is often assumed to be rigid, thereby obscuring the interaction between the solid and the fluid. Since polymeric solids in diffusion situations undergo large deformations [3,4], an improved theory is needed. A general theory of interacting continua, i.e. mixtures, based on modern continuum mechanics which would be applicable to such diffusion problems, has been proposed. A presentation of the theory as it stands today can be found in the review articles by Bowen [5] and Atkin and Craine [6].

In the applications of the theory to date, (Crochet and Naghdi[7], Mills and Steel[8], Atkin [9]) constitutive equations having a general form of free energy function and unspecified material dynamical parameters are employed. Results are presented in analytic form so that solution details which can be related qualitatively or quantitatively to experimental results are lacking. Consequently, there is only a general picture of the behavior predicted by the theory of interacting continua.

The purpose of this investigation is to provide detailed solutions of problems involving the diffusion of a fluid through a non-linear elastic solid using a constitutive equation based on realistic material properties. In Section 2, the notation and kinematic quantities are defined and the relevant field equations are presented. Restrictions based on the Clausius-Duhem inequality for the constitutive equations of a mixture of an isotropic non-linear elastic solid and a Newtonian fluid are presented in Section 3. A specific form of the Helmholtz free energy function, developed from kinetic theory (Treloar[10]), is introduced and the corresponding reductions in the general constitutive equation are obtained in Section 4.

In considering the swelling of an homogeneous body, it is not possible to determine a unique swollen state if only the total stress is specified. However, if the body is assumed to be in a saturated equilibrium state, one can gainfully employ the Flory-Huggins equation (Treloar[10]) to determine this unique swollen condition. This equation is introduced in Section 5.

The analysis in Sections 3 and 4 are for a mixture of an isotropic non-linearly elastic solid and a Newtonian fluid. In order to reduce the number of unknown material moduli and hence simplify the computational aspects of our investigations in the remainder of the analysis, we restrict out attention to a mixture of a non-linear elastic solid and an ideal fluid. The corresponding solutions for the more general case of a Newtonian fluid will be done in a separate work. 
Sections 6 and 7 treat the pressure-induced steady state diffusion of an ideal fluid through a uniformly stretched isotropic non-linear elastic layer. Section 8 treats the diffusion of an ideal fluid through a non-linear elastic hollow sphere. Each problem is governed by a non-linear differential equation having non-linear algebraic boundary conditions for which numerical solutions are obtained. In the first example comparison of numerical and experimental results leads to the determination of a material dynamic parameter and the verification of a computed ceiling flux. The layer problem is also solved using a modified Fick's law, which contains one constant to be determined by comparison with experimental results.

\section{NOTATION. BASIC EQUATIONS}

A mixture of two continua, which are in motion relative to each other, is considered. The relative motion is caused by fluid $S_{2}$ diffusing through a solid $S_{1}$. At an arbitrary time $t$, it is assumed that at each place in the region of space occupied by the mixture two particles are situated, one belonging to each constituent. Kinematics and balance equations must be stated for each constituent.

\section{Kinematics and notation}

Let the motion of the mixture be referred to a fixed system of rectangular Cartesian coordinates. Let $\mathrm{X}$ and $\mathrm{Y}$ denote reference positions for typical particles of $S_{1}$ and $S_{2}$, respectively. Positions of these particles at time $t$ are denoted, respectively, by

$$
\mathbf{x}=\chi_{1}(\mathbf{X}, t), \quad \mathbf{y}=\chi_{2}(\mathbf{Y}, t) .
$$

We assume that these motions are one-to-one, continuous, and invertible and that the bodies under consideration occupy the same position at time $t$.

Components of various kinematic quantities associated with $S_{1}$ and $S_{2}$ at point $\mathbf{x}=\mathbf{y}$ are denoted as follows

\begin{tabular}{|c|c|c|}
\hline Velocity vector & $\begin{array}{c}S_{1} \\
\mathbf{u}=\frac{\mathrm{d} \boldsymbol{\chi}_{1}}{\mathrm{~d} t}\end{array}$ & $\begin{array}{c}S_{2} \\
\mathbf{v}=\frac{\mathrm{d} \boldsymbol{X}_{2}}{\mathrm{~d} t} .\end{array}$ \\
\hline Acceleration vector & f & g. \\
\hline Velocity gradient tensor & $\mathbf{L}$ & M. \\
\hline $\begin{array}{l}\text { Rate of deformation } \\
\text { tensor }\end{array}$ & D & G. \\
\hline Voritcity tensor & $\Gamma$ & $\Lambda$. \\
\hline
\end{tabular}

The deformation gradient tensor for the solid $S_{1}$ is $\mathbf{F}=\partial_{\mathbf{x}} / \partial \mathbf{X}$. The densities of $S_{1}$ and $S_{2}$ at time $t$, measured per unit volume of the mixture, are $\rho_{1}$ and $\rho_{2}$, respectively. The mean velocity of the mixture and the total density of the mixture are defined respectively by

$$
\begin{aligned}
& \rho \mathbf{w}=\rho_{1} \mathbf{u}+\rho_{2} \mathbf{v}, \\
& \rho=\rho_{1}+\rho_{2} .
\end{aligned}
$$

\section{Basic equations}

In this section we review briefly the balance laws for mixtures which will be employed time and again during the course of this analysis.

(i) Balance of mass (continuity equation)

We assume throughout that there is no process of interconversion of masses of $S_{1}$ and $S_{2}$. The appropriate form of the mass balance equation for the solid is

$$
\rho_{1} \operatorname{det} \mathbf{F}=\rho_{10},
$$


where $\rho_{10}$ is the mass density of the solid before forming the mixture. The appropriate equation for the fluid is $\nmid$

$$
\frac{\partial \rho_{2}}{\partial t}+\operatorname{div}\left(\rho_{2} \mathbf{v}\right)=\mathbf{0}
$$

(ii) Balance of linear momentum

It is assumed that constituents $S_{1}$ and $S_{2}$ each has a partial stress tensors for $S_{1}$ and $S_{2}$, respectively, $\boldsymbol{\sigma}+\boldsymbol{\pi}$ be the total stress tensor, and $\mathbf{b}$ be the diffusive body forces vector. If there are no external body forces, then the equations of motion for $S_{1}$ and $S_{2}$, respectively reduce to

$$
\begin{aligned}
& \operatorname{div} \boldsymbol{\sigma}-\mathbf{b}=\rho_{1} \mathbf{f}, \\
& \operatorname{div} \boldsymbol{\pi}+\mathbf{b}=\rho_{2} \mathbf{g} .
\end{aligned}
$$

(iii) Balance of moment of momentum

This condition states that the total stress tensor is symmetric, i.e.

$$
\boldsymbol{\sigma}+\boldsymbol{\pi}=\boldsymbol{\sigma}^{T}+\boldsymbol{\pi}^{T}
$$

but that the partial stresses are not necessarily symmetric.

(iv) Entropy production irequality

Let $U_{i}, \eta_{i}$ and $A_{i}$ denote, respectively, the internal energy, entropy and Helmholtz free energy function, all per unit mass of $S_{i}, i=1,2$. If $S_{1}$ and $S_{2}$ are assumed to have the same temperature $T>0$, then let

$$
\begin{aligned}
& \rho \eta=\rho_{1} \eta_{1}+\rho_{2} \eta_{2}, \\
& A_{\alpha}=U_{\alpha}-T \eta_{\alpha}(\alpha=1,2), \\
& \rho A=\rho_{1} A_{1}+\rho_{2} A_{2}=\rho(U-T \eta) .
\end{aligned}
$$

The assumed form of the Clausius-Duhem inequality for the mixture is

$$
\rho T \frac{D \eta}{D t}+T \psi-\rho r+T \operatorname{div}\left(\frac{\mathbf{q}}{T}\right) \geq 0
$$

where

where

$$
\begin{aligned}
\psi & =\operatorname{div}\left(\rho_{1} \eta_{1} \mathbf{u}^{1}+\rho_{2} \eta_{2} \mathbf{v}^{1}\right) \\
\mathbf{u}^{1} & =\mathbf{u}-\mathbf{w} \\
\mathbf{v}^{1} & =\mathbf{v}-\mathbf{w} .
\end{aligned}
$$

The radiant heat supply $r$ is defined through

$$
\rho r=\rho_{1} r_{1}+\rho_{2} r_{2}
$$

where $r_{1}$ and $r_{2}$ are the radiant heating associated with $S_{1}$ and $S_{2}$ respectively. At this point we note[12] that by setting

$$
\begin{aligned}
& \mathbf{b}=\operatorname{grad} \phi_{1}+\overline{\mathbf{b}}=-\operatorname{grad} \phi_{2}+\overline{\mathbf{b}} \\
& \boldsymbol{\sigma}=\phi_{1} \mathbf{l}+\overline{\boldsymbol{\sigma}} \\
& \boldsymbol{\pi}=\phi_{2} \mathbf{l}+\overline{\boldsymbol{\pi}}
\end{aligned}
$$

thet $\Omega$ and $\Omega$, denote the reference configuration and the configuration of the body at time $t$, respectively. For a function defined on $\Omega \times \mathbf{R}$ and $\Omega_{t} \times \mathbf{R}$, we use $\nabla$ and grad to represent the partial derivative with respect to $\Omega$ and $\Omega_{t}$,
respectively. Also we denote by () and ()$_{t}$ the partial derivative with respect to $\mathbf{R}$. The divergence operator related to grad is denoted by div. 
where

$$
\phi_{1}=\rho_{1}\left(A_{1}-A\right), \quad \phi_{2}=\rho_{2}\left(A_{2}-A\right), \quad \phi_{1}+\phi_{2}=0,
$$

eqns (5)-(7) become div $\overline{\boldsymbol{\sigma}}-\overline{\mathbf{b}}=\rho_{\mid} \mathbf{f}$

$$
\begin{aligned}
\operatorname{div} \overline{\boldsymbol{\pi}}+\overline{\mathbf{b}} & =\rho_{2} \mathbf{g} \\
\overline{\boldsymbol{\sigma}}+\overline{\boldsymbol{\pi}} & =\overline{\boldsymbol{\sigma}}+\overline{\boldsymbol{\pi}}
\end{aligned}
$$

\section{(v) Surface conditions}

Let $\mathbf{t}$ and $\mathbf{p}$ be surface traction vectors taken by $S_{1}$ and $S_{2}$, respectively, at a surface element whose unit outer normal is $\mathbf{n}$. The surface conditions are

$$
\mathbf{t}=\sigma^{T} \mathbf{n}, \quad \mathbf{p}=\pi^{T} \mathbf{n}
$$

\section{CONSTITUTIVE EQUATION}

\section{Incompressibility condition}

The concept of incompressibility as introduced by Mills[13] for a binary mixture of Newtonian fluids can be used for any type of non-interconverting constituents.

We assume that $S_{1}$ is an incompressible elastic solid and $S_{2}$ is an incompressible Newtonian fluid. When separate, let $S_{1}$ and $S_{2}$ have constant densities $\rho_{10}$ and $\rho_{20}$, respectively. We shall assume that the motion of the mixture is such that it meets the following combinational volumetric law, namely the volumes of the constituents are additive. Their current densities $\rho_{1}$ and $\rho_{2}$, per unit volume of the mixture, then define the total density of the mixture by eqn (2).

Under this assumption Mills[14] has shown that the current densities satisfy the following equations, where the latter two were obtained by combining eqns (2) and (11)

$$
\begin{gathered}
\frac{\rho_{1}}{\rho_{10}}+\frac{\rho_{2}}{\rho_{20}}=1, \\
\rho_{1}=\frac{\rho_{10}\left(\rho_{20}-\rho\right)}{\rho_{20}-\rho_{10}}, \\
\rho_{2}=\frac{\rho_{20}\left(\rho-\rho_{10}\right)}{\rho_{20}-\rho_{10}} .
\end{gathered}
$$

Equation (11) imposes a constraint on the motions of the continua which leads to the introduction of an indeterminate scalar into the constitutive equations.

\section{Constitutive assumptions}

We need constitutive assumptions for $A, \eta, \overline{\mathbf{b}}, \overline{\boldsymbol{\sigma}}, \bar{\pi}, \phi_{1}, \phi_{2}$ and $\mathbf{q}$ to characterize the type of fluid and solid which interact. Since we consider a mixture of an elastic solid and a Newtonian fluid, we shall assume that all the constitutive functions depend on the following variables.

$$
\mathbf{F}, \rho_{2}, \nabla \mathbf{F}, \operatorname{grad} \rho_{2}, T, \operatorname{grad} \mathbf{T}, \mathbf{u}, \mathbf{v}, \mathbf{L} \text { and } \mathbf{M} \text {. }
$$

Following Crochet and Naghdi[7], we write the partial stresses and diffusive force as sums of a statical and a dynamical part, i.e.

$$
\begin{aligned}
\overline{\boldsymbol{\sigma}} & =\overline{\boldsymbol{\sigma}}^{s}+\overline{\boldsymbol{\sigma}}^{d}, \\
\overline{\boldsymbol{\pi}} & =\overline{\boldsymbol{\pi}}^{s}+\overline{\boldsymbol{\pi}}^{d}, \\
\overline{\mathbf{b}} & =\overline{\mathbf{b}}^{s}+\overline{\mathbf{b}}^{d},
\end{aligned}
$$

where $\overline{\boldsymbol{\sigma}}^{s}, \overline{\boldsymbol{\pi}}^{s}$ and $\overline{\mathbf{b}}^{s}$ depend on the statical variables and $\overline{\boldsymbol{\sigma}}^{d}, \overline{\boldsymbol{\pi}}, \overline{\mathbf{b}}^{d}$ together with $A, \eta, \phi_{\alpha}$ and the heat flux vector depend on all the variables. At this juncture it would be more convenient for the purposes of presentation to resort to the usual Cartesian index notation. Based on the 
energy balance law and the application of the Clausius-Duhem inequality, a procedure which is by now standard leads to the following relations

$$
\begin{gathered}
\eta==\frac{\partial A}{\partial T} \\
\bar{\sigma}_{k i}^{s}=\rho \frac{\partial A}{\partial F_{i j}} F_{k j}-p \frac{\rho_{1}}{\rho_{10}} \delta_{k i}, \\
\bar{\pi}_{k i}^{s}==\rho_{2} \frac{\partial A}{\partial \rho_{2}} \delta_{k i}-p \frac{\rho_{2}}{\rho_{20}} \delta_{k i}, \\
\bar{b}_{k}^{s}=-\rho_{2} \frac{\partial F_{i j}}{\partial x_{k}} \frac{\partial A}{\partial F_{i j}}+\rho_{1} \frac{\partial A}{\partial \rho_{2}} \frac{\partial \rho_{2}}{\partial x_{k}}-\frac{p}{\rho_{10}} \frac{\partial \rho_{1}}{\partial x_{k}},
\end{gathered}
$$

where $A$ reduces to

$$
A=A\left(F_{i j}, \rho_{2}, T\right),
$$

and $p$ is an indeterminate scalar arising from the use of the constraint eqn (11). The dynamic parts of the partial stress, and diffusive body force satisfy the reduced entropy inequality,

$$
\begin{aligned}
\bar{\sigma}_{(k i)}^{d} d_{i k}+\bar{\pi}_{(k i)}^{d} f_{i k}+\bar{\sigma}_{[k i]}^{d}\left(\Gamma_{i k}-\Lambda_{i k}\right)+ & \bar{b}_{k}^{d}\left(u_{k}-v_{k}\right)-\frac{1}{T} \\
& \times\left[q_{k}+T\left(\rho_{1} \eta_{1}\left(u_{k}-w_{k}\right)+\rho_{2} \eta_{2}\left(v_{k}-w_{k}\right)\right)\right] \frac{\partial T}{\partial x_{k}} \geq 0,
\end{aligned}
$$

where ( ) or [ ] around subscripts denote the symmetric and skew symmetric parts of the tensors, respectively.

Following arguments based on the restrictions due to the principle of material objectivity, as presented in [7], we conclude that the constitutive functions can depend upon the velocities of the constituents only through the relative velocity $u_{i}-v_{i}$, upon the velocity gradient only through rate of deformation tensors $f_{i j}$ and $d_{i j}$ and the relative vorticity tensor $\Gamma_{i j}-\Lambda_{i j}$, and upon the deformation gradient only through $B_{i j}=F_{k i} \cdot F_{k j}$. If we assume both solid and fluid are initially isotropic with a center of symmetry, then the constitutive functions depend on $F_{i j}$ through $C_{i j} \cdot F_{i k}$.

We assume that the dynamical parts of the partial stresses and diffusive force depend linearly on the dynamical variables

$$
\begin{aligned}
\bar{\sigma}_{(i j)}^{d} & =\gamma 1 d_{k k} \delta_{i j}+2 \mu_{1} d_{i j}+\gamma_{2} f_{k k} \delta_{i j}+2 \mu_{2} f_{i j}, \\
\bar{\pi}_{(i j)}^{d} & =\gamma_{3} d_{k k} \delta_{i j}+2 \mu_{3} d_{i j}+\gamma_{4} f_{k k} \delta_{i j}+2 \mu_{4} f_{i j}, \\
\bar{\sigma}_{[i j]}^{d} & =-\bar{\pi}_{[i j]}^{d}=-c_{1}\left(\Gamma_{i j}-\Lambda_{i j}\right), \\
\bar{b}_{k}^{b} & =c_{2}\left(u_{k}-v_{k}\right) .
\end{aligned}
$$

All the coefficients are functions of $\rho_{1}, \rho_{2}$ and $T$.

It then follows from (15) that

$$
\begin{gathered}
\mu_{1} \geq 0, \quad \gamma_{1}+\frac{2}{3} \mu_{1} \geq 0, \\
\mu_{4} \geq 0, \quad \gamma_{4}+\frac{2}{3} \mu_{4} \geq 0, \\
\left(\mu_{3}+\mu_{2}\right)^{2} \leq 4 \mu_{1} \mu_{4}, \\
{\left[\left(\gamma_{2}+\gamma_{3}\right)+\frac{2}{3}\left(\mu_{2}+\mu_{3}\right)\right]^{2} \leq 4\left(\gamma_{1}+\frac{2}{3} \mu_{1}\right)\left(\gamma_{3}+\frac{2}{3} \mu_{3}\right),} \\
c_{1} \geq 0, \quad c_{2} \geq 0 .
\end{gathered}
$$


Under the assumption of isotropy, the free energy function $A$ can be written in terms of an integrity basis for $C_{i j}$ as

$$
A=A\left(I_{1}, I_{2}, I_{3}, \rho_{2}, T\right)
$$

where

$$
I_{1}=C_{i i}, I_{2}=\frac{1}{2}\left(I_{1}^{2}-C_{i k} C_{i k}\right), \quad I_{3}=\operatorname{det}\left|C_{i j}\right|
$$

Note that eqns (3), (11) and (18)

$$
I_{3}^{-(1 / 2)}=1-\frac{\rho_{2}}{\rho_{20}}
$$

so that $A$ reduces to

$$
A=A\left(I_{1}, I_{2}, \rho_{2}, T\right) .
$$

Combining (8), (13), (14), (16), (18) and (20), we obtain expressions for the partial stresses

$$
\begin{aligned}
\sigma_{k i}= & \delta_{k i} \phi_{1}+\bar{\sigma}_{k i}^{s}+\bar{\sigma}_{k i}^{d} \\
= & \delta_{k i} \phi_{1}-p \frac{\rho_{1}}{\rho_{10}} \delta_{k i}+2 \rho\left[\left(\frac{\partial A}{\partial I_{1}}+I_{1} \frac{\partial A}{\partial I_{2}}\right) C_{k i}-\frac{\partial A}{\partial I_{2}} C_{k m} C_{m i}\right] \\
& +\gamma_{1} d_{p p} \delta_{k i}+2 \mu_{1} d_{k i}+\gamma_{2} f_{p p} \delta_{k i}+2 \mu_{2} f_{k i}-c_{1}\left(\Gamma_{k i}-\Lambda_{k i}\right), \\
\pi_{k i}= & -\delta_{k i} \phi_{1}+\bar{\pi}_{k i}^{s}+\bar{p}_{k i}^{d} \\
= & -\delta_{k i} \phi_{1}-p \frac{\rho_{2}}{\rho^{20}} \delta_{k i}-\rho \rho_{2} \frac{\partial A}{\partial \rho_{2}} \delta_{k i}+\gamma_{3} d_{p p} \delta_{k i}+2 \mu_{3} d_{k i} \\
& +\gamma_{4} f_{p p} \delta_{k i}+2 \mu_{4} f_{k i}+\beta \frac{\rho_{1}}{\rho_{10}} \frac{\rho_{2}}{\rho_{20}}\left(\Gamma_{k i}-\Lambda_{k i}\right) \\
b_{k}= & \frac{\partial \phi_{1}}{\partial x_{k}}+\bar{b}_{k}^{s}+\bar{b}_{k}^{d} \\
= & \frac{\partial \phi_{1}}{\partial x_{k}}-\frac{p}{\rho_{10}} \frac{\partial \rho_{1}}{\partial x_{k}}+\rho_{1} \frac{\partial A}{\partial \rho_{2}} \frac{\partial \rho_{2}}{\partial x_{k}}-\rho_{2}\left[\left(\frac{\partial A}{\partial I_{1}}+I_{1} \frac{\partial A}{\partial I_{2}}\right) \delta_{i j}\right. \\
& \left.-\frac{\partial A}{\partial I_{2}} C_{i j}\right] \frac{\partial C_{i j}}{\partial x_{k}}+\alpha \frac{\rho_{1}}{\rho_{10}} \frac{\rho_{2}}{\rho_{20}}\left(u_{k}-v_{k}\right),
\end{aligned}
$$

where $c_{1}$ and $c_{2}$ have been redefined so that they vanish with $\rho_{1}$ or $\rho_{2}$.

\section{FREE ENERGY EQUATION}

In order to evaluate the partial stresses and diffusive forces, an expression for the free energy function $A\left(I_{1}, I_{2}, I_{3}, \rho_{2}, T\right)$ is needed.

For application to problems where various swollen states may be encountered, it would seem preferable to choose the reference state as the unstrained unswollen state. We use the following free energy function for use in swelling [10],

$$
A=K\left(I_{1}-3-\frac{1}{2} \ln I_{3}\right), \quad K=\frac{1}{2} \frac{R T}{M_{c}},
$$

where $R$ is the gas constant, $T$ is absolute temperature and $M_{c}$ is the molecular weight between cross-links. 
As presented, the free energy expression $A$ does not depend explicitly on $\rho_{2}$ as in eqn (20). This is introduced by substituting from eqn (19) to give

$$
A=K\left[I_{7}-3+\ln \left(1-\frac{\rho_{2}}{\rho_{20}}\right)\right]
$$

By eqns (21)-(24)

$$
\begin{aligned}
\sigma_{k i}= & \delta_{k i} \phi_{1}-p \frac{\rho_{1}}{\rho_{10}} \delta_{i k}+2 p K C_{k i}+\gamma_{1} d_{p p} \delta_{k i}+2 \mu_{1} d_{k i} \\
& +\gamma_{2} f_{p p} \delta_{k i}+2 \mu_{2} f_{k i}-c_{1}\left(\Gamma_{k i}-\Lambda_{k i}\right), \\
\pi_{k i}= & \delta_{k i} \phi_{1}-p \frac{\rho_{2}}{\rho_{20}} \delta_{k i}+\rho \frac{\rho_{2}}{\rho_{20}} \frac{\rho_{10}}{\rho_{1}} K \delta_{k i}+\gamma_{3} d_{p p} \delta_{k i} \\
& +2 \mu_{3} d_{k i}+\gamma_{4} f_{p p} \delta_{k i}+2 \mu_{4} f_{k i}+c_{1}\left(\Gamma_{k i}-\Lambda_{k i}\right), \\
b_{k}= & \frac{\partial \phi_{1}}{\partial x_{k}}-\frac{p}{\rho_{10}} \frac{\partial \rho_{1}}{\partial x_{k}}-K \frac{\rho_{10}}{\rho_{20}} \frac{\partial \rho_{2}}{\partial x_{k}}-\rho_{2} K \frac{\partial I_{1}}{\partial x_{k}}+c_{2}\left(u_{k}-v_{k}\right) .
\end{aligned}
$$

\section{SWELLING}

In any diffusion problem the rubber will significantly increase its dimensions as fluid is absorbed. Adkins was the first to consider swelling using constitutive equations of the theory of mixtures [14]. He considered an isotropic elastic cube surrounded by an ideal fluid undergoing uniform swelling with no surface traction and no constraint of incompressibility. Two equations for the stretch ratio $\lambda$ of the block of rubber, density $\rho_{1}$ of the solid and density $\rho_{2}$ of the fluid were obtained from the expressions for total stress and conservation of mass for the solid, eqn (3). Under these conditions, it is not possible to uniquely determine the swollen condition, namely the new dimensions of the block $\lambda$ and densities $\rho_{1}$ and $\rho_{2}$. However, if the swollen state is a saturated state, then the Flory-Higgins equation $\{[10]\}$

$$
\left(\frac{t_{1}+t_{2}+t_{3}}{3}+p_{s}\right) \frac{V_{1}}{R T}=\ln (1-\nu)+\nu+\chi \nu^{2}+\frac{\rho_{10} V_{1}}{M_{c}}\left[\frac{\nu}{3}\left(\lambda_{1}^{2}+\lambda_{2}^{2}+\lambda_{3}^{2}\right)+\frac{\nu}{2}\right]
$$

where $\nu=I_{3}^{-1 / 2}=\left(\lambda_{1} \lambda_{2} \lambda_{3}\right)^{-1}$, provides the additional condition for determinacy.

In this equation, $R, T$ and $M_{c}$, were defined in Section $4, V_{1}$ is the molar volume of the fluid; $p_{s}$ is the hydrostatic pressure of the surrounding fluid, and $\chi$ is a constant depending on the particular rubber and fluid.

As a short example of its use consider the uniform swelling of a cube. Given $t_{1}=t_{2}=t_{3}$ and the hydrostatic pressure $p_{s}$, we need to find $\rho_{1}, \rho_{2}$ and $\lambda=\lambda_{1}=\lambda_{2}=\lambda_{3}$. From eqn (28) we find $\lambda$ or $\nu=1 / \lambda^{3}$. From eqn (3), $\rho_{1} \lambda^{3}=\rho_{10}$, we obtain $\rho_{1}$ and from eqn (11) we obtain $\rho_{2}$.

If swelling occurs with the constraint that $\lambda_{1}=\lambda_{2}$ are held fixed while $\lambda_{3}$ varies, the corresponding equation is

$$
\left(t_{3}+p_{s}\right) \frac{V_{1}}{R T}=\ln (1-\nu)+\nu+\chi \nu^{2}+\frac{\rho_{10} V_{1}}{M_{c}}\left(\nu \lambda_{3}^{2}-\frac{\nu}{2}\right)
$$

\section{DIFFUSION OF AN IDEAL FLUID THROUGH A NONLINEAR ELASTIC LAYER}

We examine the steady-state diffusion of an ideal fluid in the direction normal to the surface of an isotropic non-linear elastic layer. The layer is under uniform all-round extension or compression and has a pressure difference on its surfaces. Figure 1 shows the current state of the layer. We refer to the plane $z=h$ as the upstream surface and $z=0$ which is fixed to a rigid porous plate as the downstream surface. Experimental results [15] show that in these problems the relation between the flux and the pressure difference is non-linear. For the problem considered here for materials 


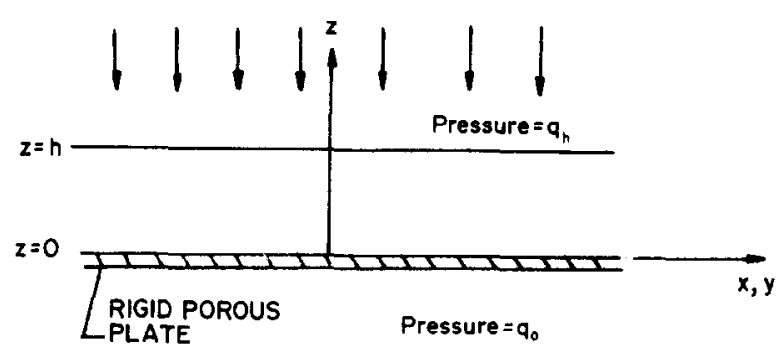

Fig. 1. Configuration for the current state of the fluid-layer mixture.

which obey the constitutive assumptions of the preceding sections, a relationship between the flux and pressure difference is found which compares favorably with available experimental results. Since we restrict our attention to the diffusion of an ideal fluid we set the following material parameters to be zero in eqns (25) and (26): $\gamma_{i}=\mu_{i}=0, i=1,2,3,4$.

The motion of the fluid and deformation of the solid are referred to a common Cartesian cordinate system. The initial position of a particle of the solid is at $(X, Y, Z)$ and its current position is $(x, y, z)$, where the two systems coincide. The plate is bounded in the undeformed state by the plane surfaces $Z=0$ and $Z=h_{0}$. Its deformation is defined by

$$
x=\lambda X, \quad y=\lambda Y, \quad z=z(Z),
$$

where $\lambda$ is a constant. Letting a prime denote differentiation with respect to the vertical coordinate $Z$, the components of the left Cauchy-Green strain tensor and its invariants are

$$
\begin{aligned}
C_{11} & =C_{22}=\lambda^{2}, \quad C_{33}=\left(z^{\prime}\right)^{2}, \quad C_{i j}=0(i \neq j), \\
I_{1} & =2 \lambda^{2}+\left(z^{\prime}\right)^{2}, \quad I_{2}=2 \lambda^{4}+\left(z^{\prime}\right)^{4}, \quad I_{3}=\lambda^{4}\left(z^{\prime}\right)^{2} .
\end{aligned}
$$

The velocity field of the fluid is given by

$$
v_{1}=v_{2}=0, \quad v_{3}=v(z)
$$

The continuity equation for the solid, eqn (3), becomes

$$
\lambda^{2} z^{\prime} \rho_{1}=\rho_{10} .
$$

In terms of the volume fraction of the solid $\nu$ and observing that $\lambda_{3}=z^{\prime}$, eqn (32) gives

$$
\nu=\left(\lambda^{2} \lambda_{3}\right)^{-1}=\left(\lambda^{2} z^{\prime}\right)^{-1}
$$

The continuity eqn (4) for the fluid implies immediately that the flux is independent of $z$

$$
\rho_{2} v=F(\text { constant })
$$

By eqns (25)-(27), (30)-(32), we obtain the following expressions for the partial stresses and diffusive force

$$
\begin{aligned}
& \sigma_{11}=\sigma_{22}=\phi_{1}-p \frac{\rho_{1}}{\rho_{10}}+2 \rho K \lambda^{2}, \\
& \sigma_{33}=2 \rho K\left(z^{\prime}\right)^{2}-p \frac{\rho_{1}}{\rho_{10}}+\phi_{1} \\
& \pi_{11}=\pi_{22}=K \rho \frac{\rho_{10}}{\rho_{20}} \frac{\rho_{2}}{\rho_{1}}-\frac{\rho_{2}}{\rho_{20}} p-\phi_{1},
\end{aligned}
$$




$$
\begin{aligned}
& \text { Applications of the theory of interacting continua } \\
& \pi_{33}=K \rho \frac{\rho_{10}}{\rho_{20}} \frac{\rho_{2}}{\rho_{1}}-\frac{\rho_{2}}{\rho_{20}} p-\phi_{1}, \\
& \sigma_{i j}=\pi_{i j}=0, \quad \text { if } i \neq j \\
& b_{1}=\frac{\partial \phi_{1}}{\partial x}, \quad b_{2}=\frac{\partial \phi_{1}}{\partial y}, \\
& b_{3}=-\frac{\rho_{10}}{\rho_{20}} K \frac{\mathrm{d} \rho_{2}}{\mathrm{~d} z}-2 \rho_{2} K z^{\prime \prime}-\frac{p}{\rho_{10}} \frac{\mathrm{d} \rho_{1}}{\mathrm{~d} z}-\alpha \frac{\rho_{1}}{\rho_{10}} \frac{\rho_{2}}{\rho_{20}} v+\frac{\mathrm{d} \phi_{1}}{\mathrm{~d} z} .
\end{aligned}
$$

The equations of motion in this problem reduce to the following forms

$$
\frac{\mathrm{d} \sigma_{33}}{\mathrm{~d} z}-b_{3}=0, \quad \frac{\mathrm{d} \pi_{33}}{\mathrm{~d} z}+b_{3}=0,
$$

where we have neglected inertial effects because the flow is assumed to be slow. Adding eqns $(41)_{1}$ and $(41)_{2}$ one obtains that

$$
\sigma_{33}+\pi_{33}=N \quad \text { (constant), }
$$

i.e. the total stress vector on planes perpendicular to the $z$ direction is independent of $z$. Substituting eqns (36) and (40) into the first of eqns (41), we get

$$
2 K \rho^{\prime}\left(z^{\prime}\right)^{2}+2 K\left(2 \rho+\rho_{2}\right) z^{\prime} z^{\prime \prime}-p^{\prime} \frac{\rho_{1}}{\rho_{10}}+\frac{\rho_{10}}{\rho_{20}} K \rho_{2}^{\prime}+\alpha \frac{\rho_{1}}{\rho_{10}} \frac{\rho_{2}}{\rho_{20}} v z^{\prime}=0
$$

Substituting eqns (36) and (38) into eqn (42), we get

$$
2 \rho K\left(z^{\prime}\right)^{2}+K \rho \frac{\rho_{10} \rho_{2}}{\rho_{20} \rho_{1}}-p=N
$$

Note that eqn (44) contains the unknown scalar $p$ explicitly. Equations (32), (34), (43) and (44) are four equations for unknowns $\rho, \rho_{1}, \rho_{2}, p, z, v$ and $F$.

Since we are interested in the distribution of the fluid along the thickness of the plate, we write $\rho$ and $\rho_{1}$ in terms of $\rho_{2}$ using eqn (12) as follows

$$
\rho_{1}=\bar{\rho}\left(\rho_{20}-\rho_{2}\right), \quad \rho=\rho_{10}{ }^{-} \rho^{*} \rho_{2},
$$

where

$$
\bar{\rho}=\frac{\rho_{10}}{\rho_{20}}, \quad \rho^{*}=\frac{\rho_{10}-\rho_{20}}{\rho_{20}} .
$$

Next, by eqns (32) and (11), we get $\rho_{2}, \rho_{2}^{\prime}$, and $v^{\prime}$ in terms of $z(Z)$, i.e.

$$
\begin{aligned}
& \rho_{2}=\frac{\rho_{20}}{\lambda^{2} z^{\prime}}\left(\chi^{2} z^{\prime}-1\right), \quad \rho_{2}^{\prime}=\rho_{20} \frac{z^{\prime \prime}}{\lambda^{2}\left(z^{\prime}\right)^{2}} \\
& v=-\frac{F \rho_{2}^{\prime}}{\rho_{2}^{2}}=-\frac{F z^{\prime \prime} \lambda^{2}}{\rho_{20}\left(\lambda^{2} z^{\prime}-1\right)^{2}} .
\end{aligned}
$$

Substituting eqns (45)-(47) into eqn (43), we get

$$
\begin{aligned}
2 K \rho * \frac{\rho_{20}}{\lambda^{2}} z^{\prime \prime} & +2 K\left[2 \rho_{10}+\left(1-2 \rho^{*}\right)\left(1-\frac{1}{\lambda^{2} z^{\prime}}\right) \rho_{20}\right] z^{\prime} z^{\prime \prime} \\
& -p^{\prime} \frac{1}{\lambda^{2} z^{\prime}}+\bar{\rho} K \rho_{20} \frac{z^{\prime \prime}}{\lambda^{2} z^{\prime 2}}+\frac{\alpha F}{\rho_{20} \lambda^{2}}=0 .
\end{aligned}
$$


Combining eqns (44)-(48), we obtain an expression for $p$

$$
p=K\left(\rho_{10}-\rho^{*} \rho_{20} \frac{\lambda^{2} z^{\prime}-1}{\lambda^{2} z^{\prime}}\right)\left(2\left(z^{\prime}\right)^{2}+\lambda^{2} z^{\prime}-1\right)-N .
$$

Substituting $p$ from eqn (50) into eqn (49) and introducing the non-dimensionalization

$$
\bar{z}=\frac{z}{h_{0}}, \quad \bar{Z}=\frac{Z}{h_{0}}, \quad \bar{z}^{\prime}=\frac{\mathrm{d} \bar{z}}{\mathrm{~d} \bar{Z}}, \quad \bar{z}^{\prime \prime}=\frac{\mathrm{d}^{2} \bar{z}}{\mathrm{~d} \bar{Z}^{2}}
$$

we obtain a non-linear second order ordinary differential equation for $z(Z)$.

$$
\begin{aligned}
\bar{z}= & -\frac{\alpha F}{\rho_{20}^{2} K} \frac{h_{0}}{\lambda^{2}}\left\{\frac{2 \rho^{*}}{\lambda^{2}}+2 \bar{z}^{\prime}\left[2 \bar{\rho}+\left(1-2 \rho^{*}\right) \frac{\lambda^{2} \bar{z}^{\prime}-1}{\lambda^{2} \bar{z}^{\prime}}\right]\right. \\
& -\frac{1}{\lambda^{2} \bar{z}^{\prime}}\left[\left(4 \bar{z}^{\prime}+\lambda^{2}\right)\left(\bar{\rho}-\rho^{*} \frac{\lambda^{2} \bar{z}-1}{\lambda^{2} \bar{z}^{\prime}}\right)-\left(2\left(\bar{z}^{\prime}\right)^{2}+\lambda^{2} \bar{z}^{\prime}-1\right)\right. \\
& \left.\left.\cdot \frac{\rho^{*}}{\lambda^{2}\left(\bar{z}^{\prime}\right) 2}\right]+\bar{\rho} \frac{1}{\lambda^{2} \bar{z}^{\prime}}\right\}^{-1} \cdot
\end{aligned}
$$

We solve the above equation numerically.

In eqn (51) the stretch ratio in the plane of the layer $\lambda$ can be prescribed. If the fluid and the solid are chosen, then $\rho_{10}, \rho_{20}$, and hence $\bar{\rho}$ and $\rho^{*}$ are known, but the constant $\alpha$, which appears in the dynamic part of $b_{i}$ in eqn (27) and depends on the constituents of the mixture, is not. In evaluating $K$, which appears in the free energy function $A$ in eqn (24), the gas constant $R$ and absolute temperature $T$ are well known physical quantities. According to [10], $M_{c}$, the molecular weight of rubber between cross-links, can be found by many experimental methods. Paul[15] indicated one method to determine the mole of cross-links per unit volume, which can be used to calculate $M_{i}$, because $M_{c}$ equals the density of rubber/moles per unit volume. As for $\alpha$, there are no existing data which we can use. For this reason, we will compute solutions for different values of $\alpha$ and then compare with experimental results. The details of how $\alpha$ is determined will be discussed later.

If upstream pressure is $q_{h}$, and downstream the layer is fixed to a rigid porous plate, then the boundary conditions are

$$
\bar{z}(0)=0 \text {, }
$$

and by eqn (42)

$$
N=-q_{k}
$$

We assume that the membrane has swollen with a fixed in-plane stretch ratio. The upper surface has reached an equilibrium state satisfying the Flory-Huggins eqn (20). Under the steady diffusion process, new fluid particles enter as present fluid particles leave. It is assumed that this flow does not change the equilibrium state. Substituting $t_{3}=N=-q_{h}$ and $p_{s}=q_{h}$ into eqn (29) and using eqn (33), the following equation is obtained for $\nu(h)$, the degree of swelling at $z=h$

$$
\ln (1-\nu(h))+\nu(h)+\chi \nu^{2}(h)+\frac{\rho_{10} V_{1}}{M_{c}}\left(\frac{1}{\Lambda^{4} \nu(h)}-\frac{\nu(h)}{2}\right)=0 .
$$

Denoting the solution of eqn (54) by $\nu^{*}$, eqn (33) then gives a direct boundary conditons for the integration of eqn (51).

$$
\bar{z}(h)=\frac{1}{\lambda^{2} \nu^{*}}
$$


Numerical integration of eqn (51) gives $\bar{z}^{\prime}(\bar{Z})$. This can then be directly integrated, using boundary condition eqn (52) to give the deformation function $\bar{z}(\bar{Z})$ for the layer. By eqn (47), the variation of fluid density $\rho_{2}(\bar{Z})$ can be calculated. In order to get the difference between upstream and downstream pressures, we use the Flory-Huggins eqn (29) for the downstream surface $\bar{Z}=0$

$$
\left(-q_{n}+q_{0}\right) \frac{V_{1}}{R T}=\ln (1-\nu(0))+\nu(0)+x \nu^{2}(0)+\frac{\rho_{10} V_{1}}{M_{c}}\left(\frac{1}{\lambda^{4} \nu(0)}-\frac{\nu(0)}{2}\right) .
$$

The volume fraction of the solid in the mixture on the downstream surface $\nu(0)$ is obtained from the numerical solution for $\bar{z}^{\prime}(\bar{Z})$ and eqn (33). Equation (56) is solved for $q_{0}$, the downstream pressure.

For computational purposes, the membrane is taken as rubber and the fluid as toluene. The material constants are obtained from Paul and Ebra-Lima[15]: $\rho_{10}=0.862 \mathrm{gm} / \mathrm{c.c}, \rho_{20}=$ $0.869 \mathrm{gm} / \mathrm{c} . \mathrm{c}, \quad V_{1}=106 \mathrm{c} . \mathrm{c} / \mathrm{mole}, M_{c}=9151 \mathrm{gm} / \mathrm{mole}$ and $\chi=0.425$. The gas constant $R=$ $8.317 \times 10^{7}$ dyne-cm $/$ mole- ${ }^{\circ} \mathrm{K}$. Taking room temperature to be $30^{\circ} \mathrm{C}$, then $T=303.16^{\circ} \mathrm{K}$. The initial membrane thickness was $h_{0}=1 \mathrm{~mm}$.

Equation (51) was numerically integrated using a fourth order Runge-Kutta method. Equations (54) and (56) were solved by Newton's method. Denote $\left(\alpha F h_{0} / \rho_{20}^{2} K\right)$ by $\alpha^{*}$. Because the constant $\alpha$ is unknown, eqn (51) was solved using a number of different values for $\alpha^{*}$. As the flow is defined to be in the negative $z$-direction, eqn (34) implies $F<0$. Hence, in eqn (51), we let $\alpha^{*}=-0.5,-1.0, \ldots$ Matching, at a pressure difference $q_{h}-q_{0}$ of $100 \mathrm{psi}$ and $\lambda=1.0$, the computed flux-pressure difference curve with the experimental results obtained in [15], the value $\alpha=21.64 \mathrm{gm} / \mathrm{day}-\mathrm{cm}$ was selected. The flux-pressure difference graph shown in Fig. 2 was constructed using this value of $\alpha$.

Case 1. $\lambda=1.0$. The experimental data obtained by Paul and Ebra-Lima[15] is for this case of an unstretched layer. Solution of eqns (51) and (54) give $\nu(h)=\nu^{*}=0.3516$ and $\bar{z}^{\prime}(h)=$ $\left(\nu^{*}\right)^{-1}=2.8236$.

Case 2. $\lambda=1.1$. The corresponding boundary conditions from eqns (51) and (54) are $\nu(h)=0.32015$ and $\bar{z}^{\prime}(h)=2.5814$.

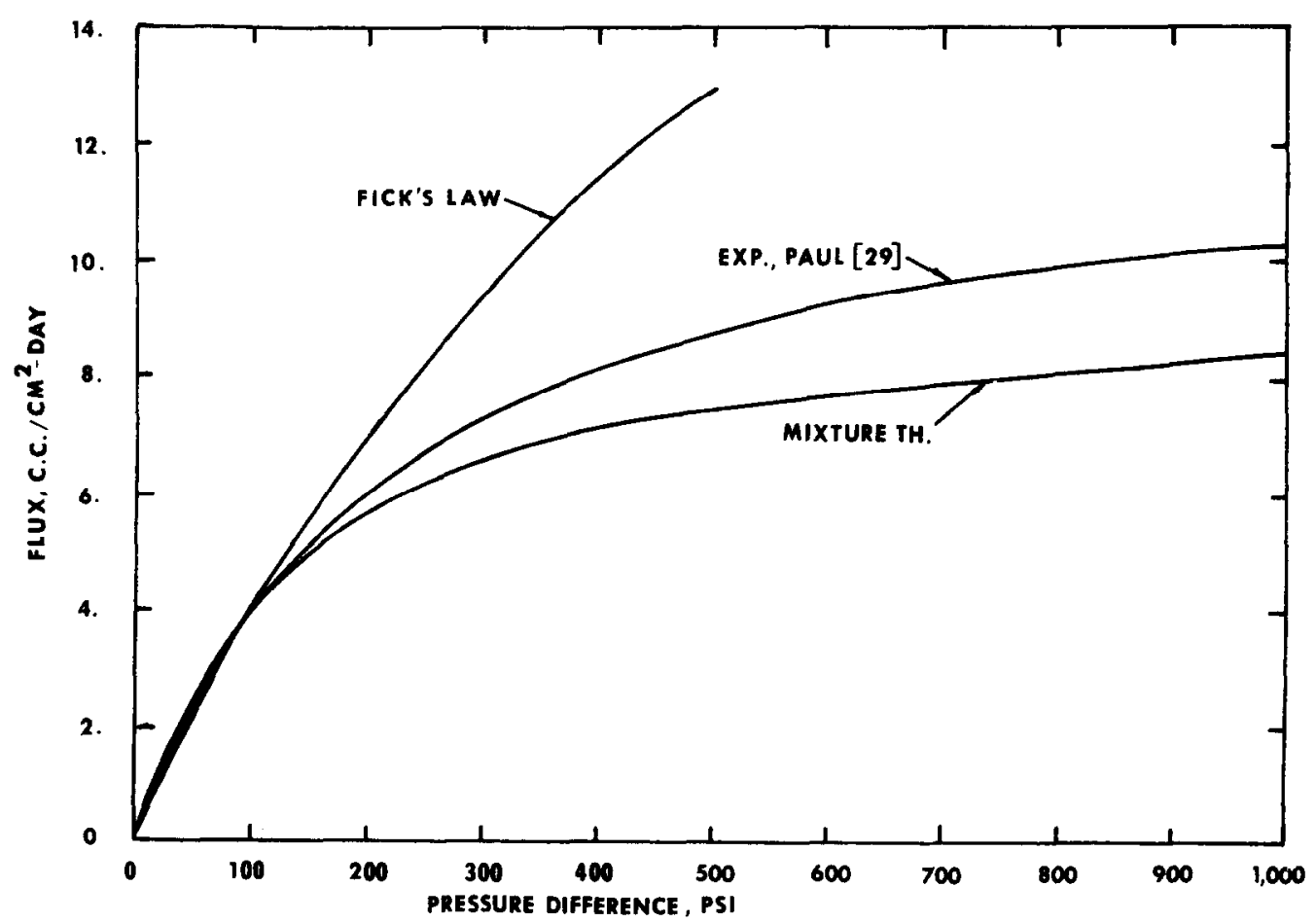

Fig. 2. Comparison of flux-pressure difference relations predicted by (a) mixture theory (b) Fick's law and (c) experimental results. 
Figure 2 shows that the results derived with $\lambda=1.0$ and the experimental results of Paul and Ebra-Lima[15] match closely. Furthermore, Paul's results indicate the existence of a ceiling flux, that is a maximum flux independent of the pressure difference. An important result of the theory is that it also predicts a ceiling flux which here is given by $\alpha^{*}=11.2$. The existence of the ceiling flux arises as follows. The value $\nu(h)$ is fixed for all values of $\alpha^{*}$. As $\alpha^{*}$ increases, $\nu(0)$ approaches the value one (Fig. 4) $\alpha^{*}$ has a finite value at the physical limit value $\nu(0)=1$, which corresponds to the absence of fluid at $Z=0$. In the swelling equation, as $\nu(0)$ approaches one, the pressure difference $q_{h}-q_{0}$ approaches infinity. Thus, flux approaches a finite value as $q_{h}-q_{0}$ becomes large.

We note from Fig. 3 that stretching increases flux through the membrane. The expression for $\alpha^{*}$ shows that for a given pressure difference the flux is inversely proportional to the layer thickness. Figure 4 shows that when the flux is small, the fluid density variation along the thickness is almost linear, as would be expected. For large flux, there is a sharp density variation near $\bar{Z}=0$ with $\rho_{2}(0)$ approaching zero, as discussed above. Figure 5 shows that the

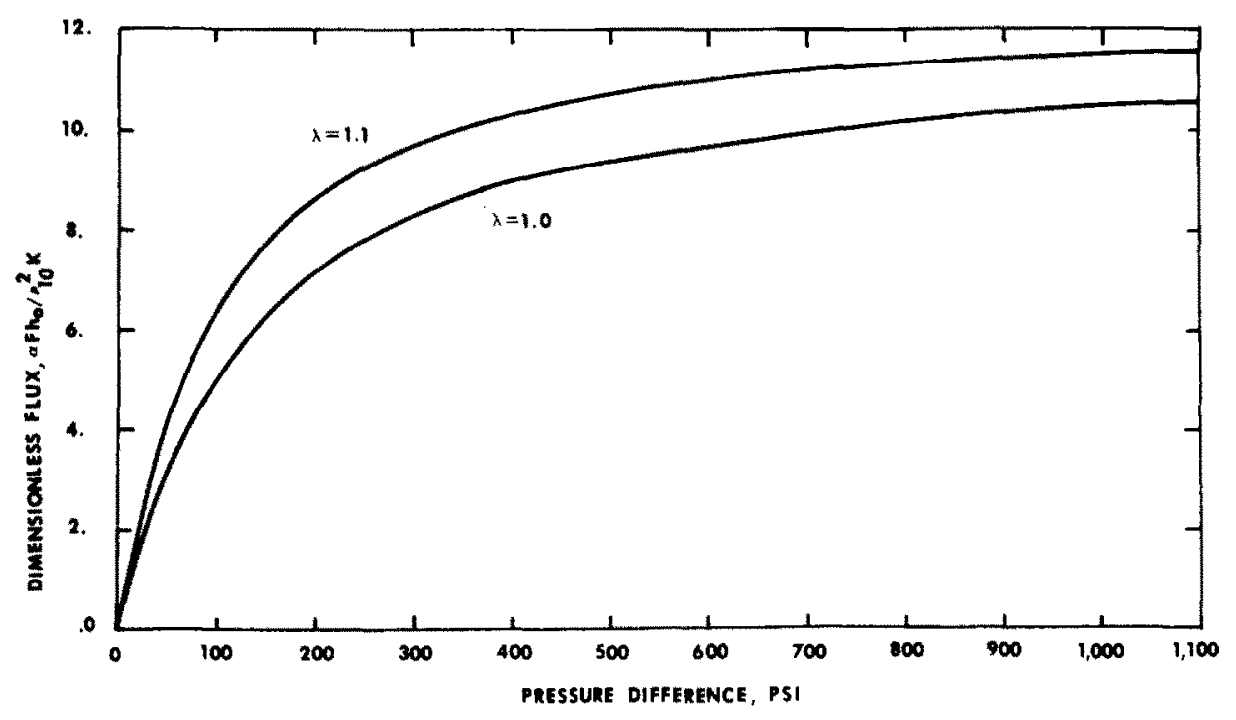

Fig. 3. Effect of stretching on the flux-pressure difference relation.

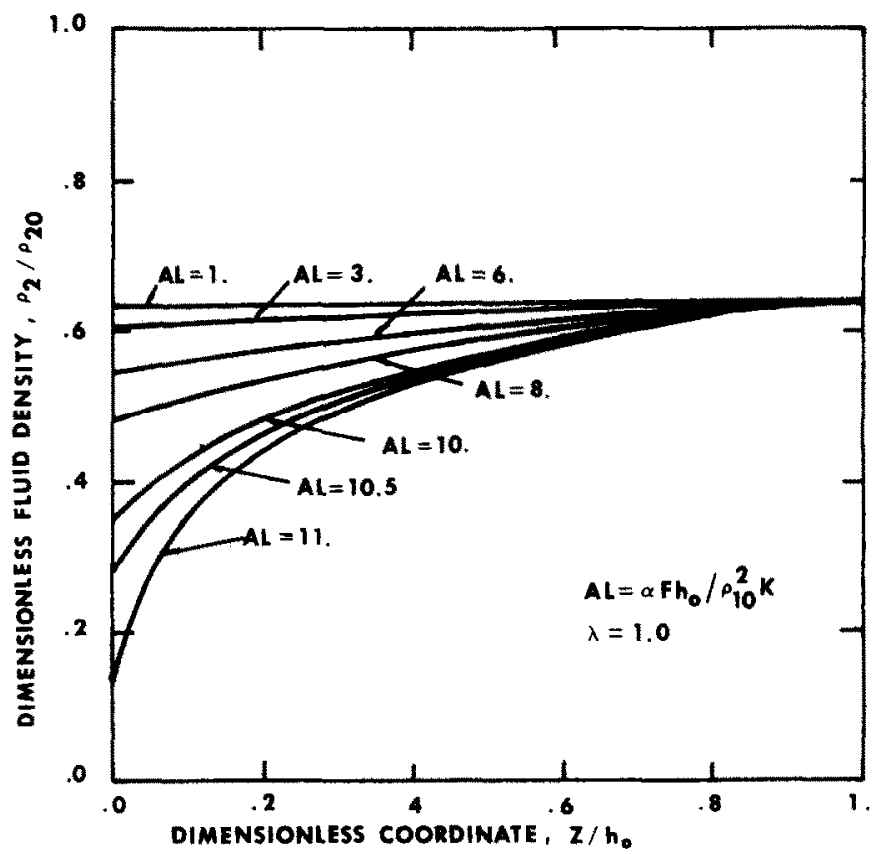

Fig. 4. Variation of fluid density through layer thickness for various values of flux. 


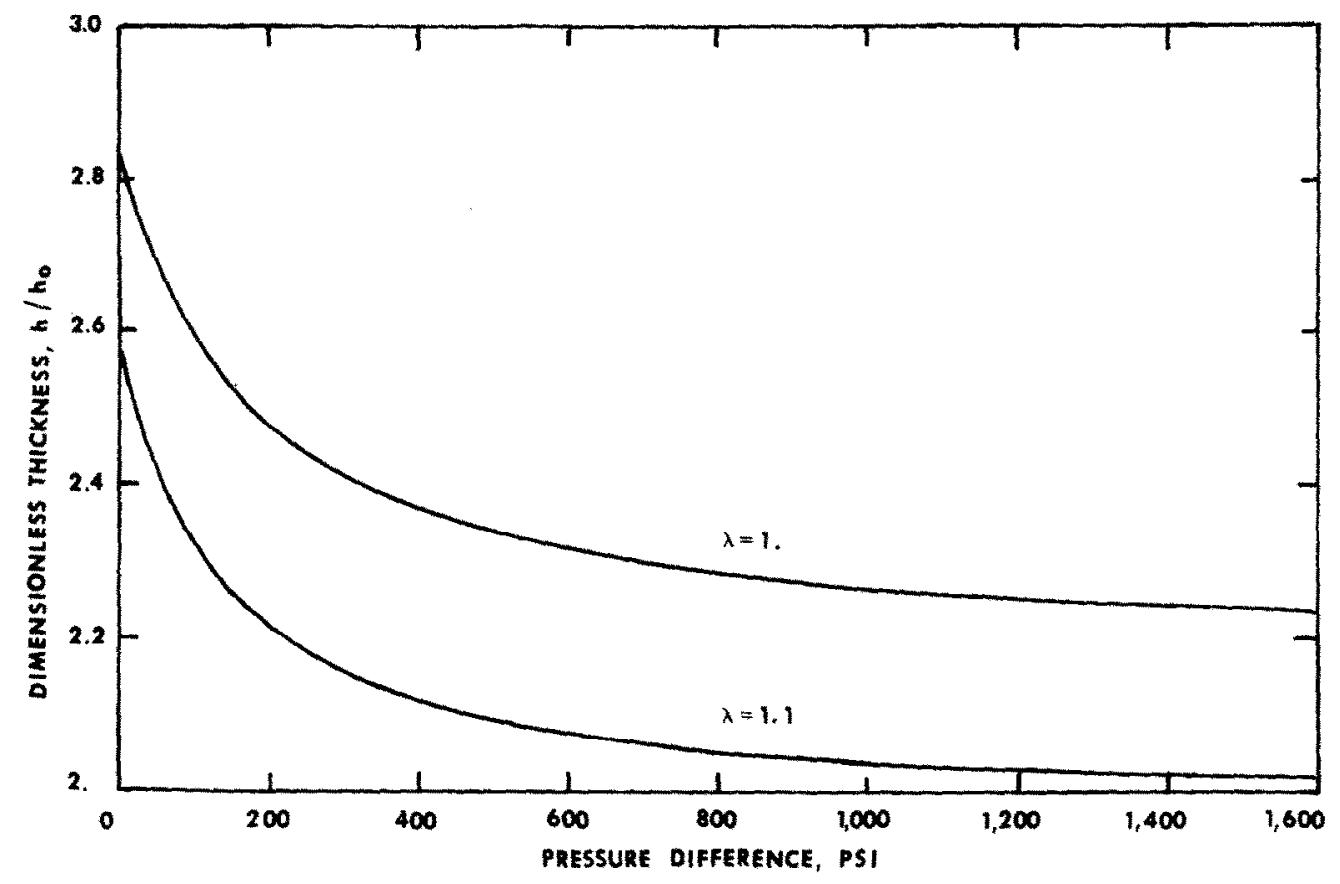

Fig. 5. Variation of deformed thickness with pressure difference.

thickness decreases as the pressure difference increases. Since Fig. 3 shows existence of a ceiling flux, these results can be interpreted as representing membranc compaction[16] associated with the limit state.

\section{ANALYSIS USING FICK'S LAW}

It would be tempting to approach diffusion problems such as the one considered here, by using the apparently analytically simpler Fick's law [1] approach which is commonly treated as the basic law or constitutive equation for the diffusion process. In such an approach the constitutive equation for the partial stresses, diffusive body force and the equations of motion are replaced by Fick's first law [1]

$$
\rho_{2}\left(v_{i}-w_{i}\right)=-\rho D \frac{\partial C_{2}}{\partial x_{i}}
$$

where $\rho_{2}$ and $\rho$ are the current mass densities of the fluid and the mixture, $C_{2}=\left(\rho_{2} / \rho\right)$ is the mass fraction of fluid in the mixture, $D>0$ is the diffusivity, $v_{i}$ is the fluid velocity vector, and $w_{i}$ is the mean velocity vector of the mixture defined in eqn (1). In fact there is a substantial body of literature in chemical engineering where such an approach is used. Of course, the use of eqn (57) as the governing equation would not be totally appropriate for the problem in question. The diffusivity $D$ in eqn (57) should be taken as some non-linear function of the deformation of the layer. However, in the absence of any rational mechanism for choosing a specific function for $D$, as a first attempt we select $D$ to be a constant. We find in this section, by carrying out an analysis based on eqn (57) that such an approach with $D$ being a constant leads to results which are at odds with those obtained from mixture theory. Of course, a diffusion theory based on a Fick's law approach can be derived as a special case of mixture theory, wherein the results obtained by using it would be compatible with those obtained from mixture theory. But in this case one would not obtain eqn (57) as the form of Fick's law with the diffusivity $D$ being a constant. In this analysis we shall not concern ourselves with obtaining a proper form of Fick's law from the more general continuum theory for interacting media. Instead, in this section we shall make a comparison between the results obtained in Section 6 and the results based on a eqn (57) with the diffusivity $D$ being a constant. In fact it is our purpose to emphasize that random generalizations of eqn (57) would not be valid. It should be possible to obtain a specific function $D$ of the deformation gradient which indeed would lead to 
a valid constitutive model. But as we mentioned earlier we have no rational means of arriving at the specific function. The best one could do is to use experimental results and curve matching procedures to determine the form of $D$.

In the present problem, using eqn (1)

$$
\begin{aligned}
& v_{1}=v_{2}=0, \quad v_{3}=v(z), \\
& w_{1}=w_{2}=0, \quad w_{3}=\frac{\rho_{2}}{\rho} v(z)=C_{2} v(z) .
\end{aligned}
$$

Equation (57) then becomes

$$
\rho_{2} v=-\frac{\rho D}{1-C_{2}} \frac{\mathrm{d} C_{2}}{\mathrm{~d} z}
$$

The conservation of mass equations for the solid and fluid must still be satisfied. By the conservation of mass equation for the fluid, eqn (34), eqn (58) becomes

$$
-\frac{\rho D}{1-C_{2}} \frac{\mathrm{d} C_{2}}{\mathrm{~d} z}=F, \quad \text { (constant). }
$$

Substituting eqn (45) in eqn (59), defining $\hat{\rho}=1 / \rho$ and integrating gives the following equation

$$
\ln \frac{\hat{\rho}}{\left(\rho^{*}+1\right)-\rho_{10} \hat{\rho}}=-\frac{F\left(\rho^{*}+1\right)}{D \rho_{10}} z+\psi
$$

where $\psi$ is the constant of integration.

The swelling eqns (54) and (56) provide boundary conditions for $\rho$ at $z=0, h$. There is no difficulty regarding the use of these equations in a Fick's law approach because the surface tractions required on the upstream and downstream surface can be found by simple force balance arguments. Thus, eqns (54) and (56) can be solved for $\nu(0)$ and $\nu(h)$. Also by eqns (45) and (46)

$$
\hat{\rho}=\left(\rho_{10}-\rho_{20}\right) \nu+\rho_{20},
$$

so that

$$
\left.\hat{\rho}\right|_{z=0, h}=\left[\rho_{20}+\left.\left(\rho_{10}-\rho_{20}\right) \nu\right|_{z=0, h}\right]^{-1} .
$$

Equations (60) and (62) give an expression for $\psi$

$$
\psi=\ln \frac{\hat{\rho}(0)}{\left(\rho^{*}+1\right)-\rho_{10} \hat{\rho}(0)},
$$

which together with eqns $(60)$ and $(62)$ yield

$$
F=\frac{D \rho_{10}}{\left(\rho^{*}+1\right) h} \ln \frac{\hat{\rho}(0)\left[\left(\rho^{*}+1\right)-\rho_{10} \hat{\rho}(h)\right]}{\hat{\rho}(h)\left[\left(\rho^{*}+1\right)-\rho_{10} \hat{\rho}(0)\right]}
$$

The swollen thickness $h$ is still unkown. Solving eqn (33) for $\bar{z}^{\prime}$ and using eqn (61), one obtains

$$
h_{0}=\int_{0}^{h} \lambda^{2} \nu(z) \mathrm{d} z=\lambda^{2} \int_{0}^{h} \frac{\rho-\rho_{20}}{\rho_{10}-\rho_{20}} \mathrm{~d} z .
$$


Solving for $\rho=\hat{\rho}^{-1}$ from eqn (60), substituting into eqn (65) and integrating, gives

$$
\frac{h_{0}\left(\rho_{10}-\rho_{20}\right)\left(1+\rho^{*}\right)}{\lambda^{2}}=\frac{D \rho_{10}}{F\left(\rho^{*}+1\right)}\left\{\exp \left[\frac{F\left(\rho^{*}+1\right)}{D \rho_{10}} h-\psi\right]-\exp (-\psi)\right\}
$$

Eliminating $F$ from eqn (66) by using eqn (64), gives

$$
\frac{h}{h_{0}}=\frac{\left(\rho_{10}-\rho_{20}\right)\left(1+\rho^{*}\right)}{\lambda^{2}}\left\{\frac{1}{c}[\exp (c-\psi)-\exp (-\psi)]\right\}^{-1},
$$

where

$$
c=\ln \frac{\hat{\rho}(0)\left[\left(\rho^{*}+1\right)-\rho_{10} \hat{\rho}(h)\right]}{\hat{\rho}(h)\left[\left(\rho^{*}+1\right)-\rho_{10} \hat{\rho}(0)\right]}
$$

Finally, substituting eqn (67) into (64), we get

$$
\frac{F}{D}=\frac{\rho_{10} \lambda^{2}}{\left(\rho^{*}+1\right)^{2} h_{0}\left(\rho_{10}-\rho_{20}\right)}[\exp (c-\psi)-\exp (-\psi)]
$$

Once $\nu(0)$ and $\nu(h)$ have been computed, constants $c$ and $\psi$ can be found from eqns (62), (63) and (68). Equations (67) and (69) give $\left(h / h_{0}\right)$ and $F / D$.

If the result calculated by eqn (69) at a pressure difference $q_{h}=q_{0}$ of 100 psi is matched with the experimental result by Paul and Ebra-Lima[15], then we find that $D=53.25 \mathrm{~cm}^{2} /$ day. Using this $D$, the variation of flux with the pressure according to Fick's law is shown in Fig. 2. This figure shows that the results obtained by using a model based on mixture theory is in much better agreement with the experimental results of Paul and Ebra-Lima[15] than the results obtained using eqn (57) in conjunction with the assumption that $D$ is a constant. We would like to emphasize that this result does not mean that a Fick's law approach cannot be used but it does imply that a Fick's law approach based on eqn (57) with $D$ being a constant would be inappropriate.

\section{PRESSUREINDUCED STEADY-STATE DIFFUSION OF AN IDEAL FLUID THROUGH A THICK SPHERICAL SHELL}

Consider the pressure induced diffusion of a fluid through a spherical shell. The inner and outer radii before deformation are taken to be 1 and $a$, respectively, assuming non-dimensionalization with respect to the inner radius has taken place. Let the inner radius of the shell after deformation be $r_{i}$ and the pressure on it be $q_{i}$ and let the outer radius be $r_{0}$ with pressure $q_{0}$.

We refer the motion of the body to a system of spherical polar coordinates. A point originally at $(R, \theta, \phi)$ is now at $(r(R), \theta, \phi)$. It is a consequence of the symmetry of the problem that the $(r, \theta, \phi)$ directions are all principal directions of stress and stretch. This implies that the only non-zero components of the strain tensor $C_{i j}$ with respect to these coordinates are $C_{11}=\lambda_{r}^{2}, C_{22}=C_{33}=\lambda_{\theta}^{2}$ where $\lambda_{r}=\mathrm{d} r / \mathrm{d} R, \lambda_{\theta}=r / R$. Then the invariants, from eqn (18), are

$$
\begin{aligned}
& I_{1}=\left(\frac{\mathrm{d} r}{\mathrm{~d} R}\right)^{2}+2\left(\frac{r}{R}\right)^{2}, \\
& I_{2}=\left(\frac{r}{R}\right)^{4}+2\left(\frac{r}{R}\right)^{4}\left(\frac{\mathrm{d} r}{\mathrm{~d} R}\right)^{2} \\
& I_{3}=\left(\frac{\mathrm{d} r}{\mathrm{~d} R}\right)^{2}\left(\frac{r}{R}\right)^{4} .
\end{aligned}
$$

The velocity vector of the fluid has components

$$
v_{1}=v(r), \quad v_{2}=v_{3}=0
$$


Let $\sigma^{i j}$ and $\pi^{i j}$ now denote the physical components with respect to spherical coordinates of the partial stress tensors of the solid and fluid, respectively. Equation (25) gives for the solid

$$
\begin{aligned}
& \sigma^{11}=\left(\phi_{1}-p \frac{\rho_{1}}{\rho_{10}}\right)+2 \rho K \lambda_{r}^{2} \\
& \sigma^{22}=\sigma^{33}=\left(\phi_{1}-p \frac{\rho_{1}}{\rho_{10}}\right)+2 \rho K \lambda_{\theta}^{2} \\
& \sigma^{i j}=0, \text { if } \quad i \neq j
\end{aligned}
$$

Equation (26) yields

$$
\begin{aligned}
& \pi^{11}=\left(\rho K \frac{\rho_{2}}{\rho_{1}} \frac{\rho_{10}}{\rho_{20}}-\frac{\rho_{2}}{\rho_{20}} p-\phi_{1}\right) \\
& \pi^{22}=\pi^{33}=\rho K \frac{\rho_{2}}{\rho_{1}} \frac{\rho_{10}}{\rho_{20}}-\frac{\rho_{2}}{\rho_{20}} p-\phi_{1} \\
& \pi^{i j}=0, \text { if } i \neq j .
\end{aligned}
$$

Let $\tilde{b_{i}}$ denote the physical components of the diffusive force in spherical coordinates. Then eqn (27) gives

$$
\begin{aligned}
\tilde{b}_{1}= & \frac{\partial \phi_{1}}{\partial r}-\frac{\rho_{10}}{\rho_{20}} K \frac{\mathrm{d} \rho_{2}}{\mathrm{~d} r}-\rho_{2} K \frac{\mathrm{d}\left(\lambda_{r}^{2}+2 \lambda_{\theta}^{2}\right)}{\mathrm{d} r}-\frac{p}{\rho_{10}} \frac{\mathrm{d} \rho_{1}}{\mathrm{~d} r} \\
& -\alpha \frac{\rho_{1}}{\rho_{10}} \frac{\rho_{2}}{\rho_{20}} v, \quad \tilde{b}_{2}=\frac{\partial \phi_{1}}{\partial \theta}, \quad \tilde{b}_{3}=\frac{\partial \phi_{1}}{\partial \phi} .
\end{aligned}
$$

The equations of motion in spherical coordinates, in the absence of the external body force, are satisfied identically in the $\phi$ and $\theta$ directions, respectively. In the $r$-direction, the equations of motion for the solid and fluid are, respectively

$$
\begin{aligned}
& \frac{\mathrm{d} \sigma^{11}}{\mathrm{~d} r}+\frac{2\left(\sigma^{11}-\sigma^{22}\right)}{r}-\tilde{b}_{1}=0, \\
& \frac{\mathrm{d} \pi^{11}}{\mathrm{~d} r}+\frac{2\left(\pi^{11}-\pi^{22}\right)}{r}+\tilde{b}_{1}=0,
\end{aligned}
$$

where the inertia term has been neglected. Adding eqns (73) and (74) we obtain

$$
\frac{\mathrm{d}\left(\sigma^{11}+\pi^{11}\right.}{\mathrm{d} r}+\frac{2\left[\left(\pi^{11}+\sigma^{11}\right)-\left(\pi^{22}+\sigma^{22}\right)\right]}{r}=0 .
$$

The conservation of mass equation for the solid is, by eqn (3)

$$
\rho_{1} \lambda_{r} \lambda_{\theta}^{2}=\rho_{10} .
$$

Also, the specific volume $\nu$ of the solid is given by

$$
\frac{\rho_{1}}{\rho_{10}}=\frac{1}{\lambda_{r} \lambda_{\theta}^{2}}=\frac{1}{\nu}
$$

The continuity equation for the fluid gives

$$
r^{2} \rho_{2} v=F,
$$

where $F$ is a constant representing mass flux through the shell. 
From eqne (2) and (11), we obtain

$$
\begin{aligned}
& \rho_{2}=\rho_{20}\left(1-\frac{1}{\lambda_{r} \lambda_{\theta}^{2}}\right), \\
& \rho=\rho_{20}+\frac{1}{\lambda_{r} \lambda_{\theta}^{2}}\left(\rho_{10}-\rho_{20}\right) .
\end{aligned}
$$

Substituting eqns (70) and (72) into eqn (73), we get

$$
\begin{aligned}
& -\frac{\rho_{1}}{\rho_{10}} \frac{\mathrm{d} \rho}{\mathrm{d} r}+\frac{\mathrm{d}\left(\rho K \lambda_{r}^{2}\right)}{\mathrm{d} r}+\frac{2}{r}\left(2 \rho K \lambda_{r}^{2}-2 \rho K \lambda_{\theta}^{2}\right)+\frac{\rho_{10}}{\rho_{20}} K \frac{\mathrm{d} \rho_{2}}{\mathrm{~d} r} \\
& +\rho_{2} K \frac{\mathrm{d}\left(\lambda_{r}^{2}+2 \lambda_{\theta}^{2}\right)}{\mathrm{d} r}+\alpha \frac{\rho_{1}}{\rho_{10}} \frac{\rho_{2}}{\rho_{20}} v=0 .
\end{aligned}
$$

Substituting eqns (70) and (71) into eqn (75), we get

$$
\left.\frac{\mathrm{d} p}{\mathrm{~d} r}=\frac{\mathrm{d}\left(2 \rho K \lambda_{r}^{2}+\rho K \frac{\rho_{2}}{\rho_{1}} \rho_{20}\right.}{\rho_{20}}\right)+\frac{2}{r}\left(2 \rho K \lambda_{r}^{2}-2 \rho K \lambda_{\theta}^{2}\right) .
$$

Equations (77)-(82) are 6 equations for $\rho, \rho_{1}, \rho_{2}, r, p$ and $v$. Substituting eqn (82) into eqn (81) in order to eliminate $p$, using eqns (77)-(82), we can eliminate $\rho, \rho_{1}, \rho_{2}, p$ and $v$ to obtain

$$
\begin{aligned}
& \frac{\mathrm{d} \lambda_{r}}{\mathrm{~d} R}\left\{2\left(1-\frac{1}{\lambda_{r} \lambda_{\theta}^{2}}\right)\left(2+\frac{\rho^{*}}{\lambda_{\theta}^{2} \lambda_{r}}\right)-\frac{1}{\lambda_{\theta}^{2} \lambda_{\theta}^{2}}\left(\lambda_{\theta}^{2}+\rho^{*} \frac{1}{\lambda^{2} \lambda_{\theta}^{2}}\right)\right. \\
& \left.+\frac{\bar{\rho}}{\lambda_{r}^{3} \lambda_{\theta}^{2}}+2\left(1-\frac{1}{\lambda_{r} \lambda_{\theta}^{2}}\right)\right\}+\frac{\mathrm{d} \lambda_{\theta}}{\mathrm{d} R}\left\{-4\left(1-\frac{1}{\lambda_{r} \lambda_{\theta}^{2}}\right) \frac{\rho^{*}}{\lambda_{\theta}^{3}}\right. \\
& \left.\quad-\frac{2}{\lambda_{r}^{2} \lambda_{\theta}^{2}}\left(\lambda_{r} \lambda_{\theta}+\frac{\rho^{*}}{\lambda_{r} \lambda_{\theta}^{3}}\right)+\frac{2 \bar{\rho}}{\lambda_{r}^{2} \lambda_{\theta}^{3}}+\frac{4 \lambda_{\theta}}{\lambda_{r}}\left(1-\frac{1}{\lambda_{r} \lambda_{\theta}^{2}}\right)\right\} \\
& +\frac{4}{R \lambda_{\theta}}\left(1-\frac{1}{\lambda_{r} \lambda_{\theta}^{2}}\right)\left(1+\rho^{*} \frac{1}{\lambda_{r} \lambda_{\theta}^{2}}\right)\left(\lambda_{r}^{2}-\lambda_{\theta}^{2}\right)+\frac{\alpha F}{K \rho_{20} R_{i} R^{2} \lambda_{r} \lambda_{\theta}^{2}}=0 .
\end{aligned}
$$

$R_{i}$ is the actual inner radius used in non-dimensionalization. A second relation between $\lambda_{r}$ and $\lambda_{\theta}$ is given by a compatibility condition

$$
\frac{\mathrm{d} \lambda_{\theta}}{\mathrm{d} R}=\frac{\lambda_{r}-\lambda_{\theta}}{R}
$$

The boundary conditions at $R=1$ are

$$
r=r_{i}, \quad \sigma^{\prime \prime}+\pi^{\prime \prime}=-q_{i}
$$

By eqns (70) and (71), this gives

$$
-p(1)+K \rho_{20}\left(1+\frac{\rho^{*}}{\lambda_{r}(1) \lambda_{\theta}^{2}(1)}\right)\left[\left(\lambda_{r}(1) \lambda_{\theta}^{2}(1)-1\right)+2 \lambda_{r}^{2}(1)\right]=-q_{i}
$$

The degree of swelling at $R=1$ is controlled by the Flory-Huggins equation, without constraint, eqn (28). Setting $p_{\mathrm{s}}=q_{i}, t_{1}=-q_{i}, t_{2}=t_{3}=\sigma^{22}+\pi^{22}$, substituting from eqns (11), (46), (70), (71), (80) and (85), gives at $R=1$

$$
\begin{gathered}
\frac{4}{3} K \rho_{10} \frac{V_{1}}{R T}\left(1+\frac{\rho^{*}}{\lambda_{r}(1) \lambda_{\theta}^{2}(1)}\right)\left(\lambda_{\theta}^{2}(1)-\lambda_{r}^{2}(1)\right)=\ln (1-\nu(1))+\nu(1) \\
+\chi \nu^{2}(1)+\frac{\rho_{10} V_{1}}{M_{c}}\left[\frac{\nu(1)}{3}\left(\lambda_{r}^{2}(1)+2 \lambda_{\rho}^{2}(1)\right)-\frac{\nu(1)}{2}\right]
\end{gathered}
$$




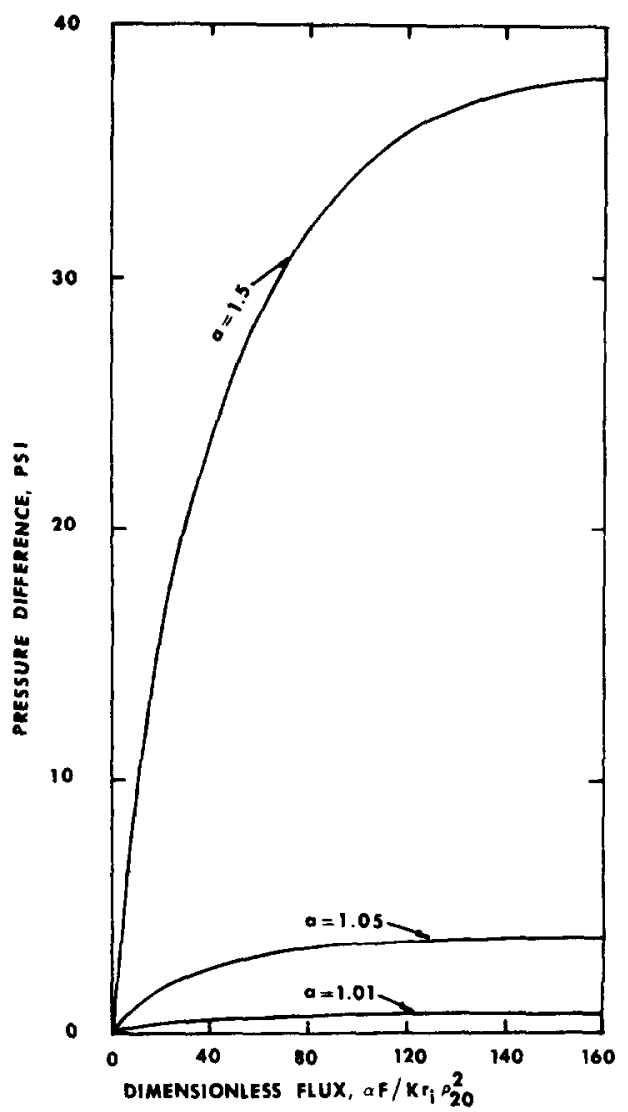

Fig. 6. Variation of flux with pressure difference for spherical shells. $a=$ undeformed outside radius/undeformed inside radius

where $v$ is defined in eqn (77). The boundary conditions at $r=a$ are

$$
r=r_{0}, \quad \sigma^{11}+\pi^{11}=-q_{0} .
$$

An equation analogous to eqn ( 85$)$ is obtained at $R=a$. To save writing, denote it by ( $85 \mathrm{a}$ ). The Flory-Huggins swelling equation at $R=a$ is obtained by evaluating all quantities in (86) at $R=a$. Denote this eqn by (86a).

The problem is now formulated at a set of two non-linear coupled ordinary first order differential eqns (83) and (84), with boundary conditions (86) and (86a).

Numerical results have been obtained for a (undeformed outside radius/undeformed inside radius) $=1.01,1.05$ and 1.5. Figure 6 shows that the pressure difference becomes almost constant at flux increases. This is in contrast to the plate response shown in Fig. 2, and apparently arises from the variation of in-plane stretch with flux. Results show that the inside and outside radii and shell thickness increase with flux. Furthermore, $\lambda_{r}, \lambda_{\theta}$ and $\rho_{2} / \rho_{20}$ vary only slightly through the shell thickness. This suggests that a membrane approximation be developed which is done in a separate work.

Acknowledgement-We wish to acknowledge several useful comments and suggestions which were made by the reviewer.

[1] B. R. BIRD, W. E. STEWART and E. N. LIGHTFOOT, Transport Phenomena. Wiley, New York (1960).

[2] A. E. SCHEIDEGGER, Hydrodynamics in Porous Media, Handbuch der Physik (Edited by S. Flugge and C. Truesdell), Vol. III/2. p. 625. Springer-Verlag, Berlin (1933).

[3] H. YASUDA, V. STAMMETTE, H. L. FRISCH and A. PETERLIN, Makromolek Chem. 73, 188 (1964).

[4] R. M. BARRER, Diffusion in and Through Solids. MacMillan, New York (1941).

[5] R. M. BOWEN, Continuum Physics (Edited by A. C. Eringen), Vol. 3. Academic Press, New York (1976).

[6] R. J. ATKIN and R. E. CRAINE, Q. J. Mech. Appl. Math. 29(2), 209 (1976). 
[7] M. J. CROCHET and P. M. NAGHDI, Int. J. Engng Sci. 4, 383 (1966).

[8] N. MILLS and T. R. STEEL, ACTA Mechanica, 9, 229 (1970).

[9] R. J. ATKIN, ZAMP, 18, 803 (1967).

[10] L. R. TRELOAR, The Physics of Rubber Elasticity. Oxford (1975).

[11] A. E. GREEN and P. M. NAGHDI, Int. J. Engng Sci. 3, 231 (1965).

[12] A. E. GREEN and P. M. NAGHDI, J. Mech. Appl. Math. 22, 427 (1969).

[13] N. MILLS, Int. J. Engng Sci. 4, 97 (1966).

[14] J. E. ADKINS, Phil. Trans. R. Soc., A, 256, 301 (1964).

[15] D. R. PAUL and D. M. EBRA-LIMA, J. Appl. Polymer Sci. 14, 2201 (1970).

[16] U. MERTEN, Desalination by Reverse Osmosis. M.I.T. Press, Cambridge, Massachusetts (1966).

(Received 5 March 1980) 